\title{
THE BOURGAIN ALGEBRA OF A NEST ALGEBRA
}

\author{
by TIMOTHY G. FEEMAN
}

(Received 25th May 1995)

\begin{abstract}
In analogy with a construction from function theory, we herein define right, left, and two-sided Bourgain algebras associated with an operator algebra $\mathcal{A}$. These algebras are defined initially in Banach space terms, using the weak-* topology on $\mathcal{A}$, and our main result is to give a completely algebraic characterization of them in the case where $\mathcal{A}$ is a nest algebra. Specifically, if $\mathcal{A}=\operatorname{alg} \mathcal{N}$ is a nest algebra, we show that each of the Bourgain algebras defined has the form $\mathcal{A}+\mathcal{X} \cap \mathcal{B}$, where $\mathcal{B}$ is the nest algebra corresponding to a certain subnest of $\mathcal{N}$. We also characterize algebraically the second-order (and higher) Bourgain algebras of a nest algebra, showing for instance that the second-order two-sided Bourgain algebra coincides with the two-sided Bourgain algebra itself in this case.
\end{abstract}

1991 Mathematics subject classification: 47D25.

Bourgain has shown ([2]) that, if $X$ is a subspace of a $C(K)$ space such that a certain set associated with $X$ coincides with $C(K)$, then $X$ has the so-called Dunford-Pettis Property (DPP). This associated set was shown to be a norm-closed algebra by Cima, et al. $([3,4])$, who labelled it the "Bourgain algebra" of $X$, and has since been studied for various spaces of functions by several authors (cf. [8, 9, 10]). In [7], this author defined an analogue of the Bourgain algebra for algebras of operators on a Hilbert space and computed this Bourgain algebra for certain examples of nest algebras as well as for the algebra of analytic Toeplitz operators on the Hardy space $H^{2}$ of the unit circle.

In this paper, we give an alternative formulation of the Bourgain algebra for an operator algebra that better reflects the non-commutativity of the operator setting and provide complete algebraic characterizations for both the earlier and new formulations of the Bourgain algebra of a nest algebra. In the last section, we characterize the second-order, and higher, Bourgain algebras of a nest algebra, showing, for instance, that the second-order (two-sided) Bourgain algebra coincides with the Bourgain algebra itself.

The author hereby expresses his gratitude to the Department of Mathematics and Statistics of Lancaster University, and especially S. C. Power, for their generous hospitality during his sabbatical visit. 


\section{Preliminaries}

In what follows, $\mathcal{H}$ will denote a separable, infinite-dimensional Hilbert space while $\mathcal{L}(\mathcal{H})$ and $\mathcal{K}$ will denote the algebra of bounded linear operators on $\mathcal{H}$ and the ideal of compact operators in $\mathcal{L}(\mathcal{H})$, respectively. All projections in $\mathcal{L}(\mathcal{H})$ are assumed to be self-adjoint and all subspaces closed. For $P$ a projection in $\mathcal{L}(\mathcal{H})$, we denote the orthogonal complement by $P^{\perp}=(1-P)$. For fixed vectors $x$ and $y$ in $\mathcal{H}$, the rankone operator $z \mapsto(z, y) x$, for $z \in \mathcal{H}$, will be denoted by $x \otimes y^{*}$. Recall that $\left\|x \otimes y^{*}\right\|=\|x\| \cdot\|y\|$.

The identification of $\mathcal{L}(\mathcal{H})$ with the dual space of the ideal of trace-class operators on $\mathcal{H}$ induces a weak-* topology on $\mathcal{L}(\mathcal{H})$ which we use throughout. Specifically, a sequence $\left\{T_{n}\right\}$ in $\mathcal{L}(\mathcal{H})$ converges to 0 in the weak-* topology if, and only if, the traces $\operatorname{tr}\left(X T_{n}\right)$ converge to 0 for every fixed trace-class operator $X$. If $y$ is a fixed vector and if $\left\{x_{n}\right\}$ is an orthonormal set, then the sequences of operators $\left\{x_{n} \otimes y^{*}\right\}$ and $\left\{y \otimes x_{n}^{*}\right\}$ converge to 0 in the weak-* topology. Indeed, if $X$ is in the trace class, then $\operatorname{tr}\left(X\left(y \otimes x_{n}^{*}\right)\right)=\left(X y, x_{n}\right) \rightarrow 0$ as $\left\{x_{n}\right\}$ is an orthonormal set. Similarly, $\operatorname{tr}\left(X\left(x_{n} \otimes y^{*}\right)\right)=\left(x_{n}, X^{*} y\right) \rightarrow 0$.

Of particular interest below are subalgebras $\mathcal{A}$ of $\mathcal{L}(\mathcal{H})$ with the property that $\mathcal{A} \cap \mathcal{K}$ is dense in $\mathcal{A}$ in the weak-* topology. In [6], such an algebra $\mathcal{A}$ is said to be local, and it is shown there that, in this case, $\mathcal{A}+\mathcal{K}$ is norm-closed.

The nest algebras form a special class of local subalgebras of $\mathcal{L}(\mathcal{H})$. Specifically, a nest is a totally ordered set of projections in $\mathcal{L}(\mathcal{H})$ which contains 0 and 1 and is closed under suprema and infima. Given a nest $\mathcal{N}$, the corresponding nest algebra, alg $\mathcal{N}$, is the algebra of operators leaving all projections in $\mathcal{N}$ invariant; that is, $\operatorname{alg} \mathcal{N}=\{T \in \mathcal{L}(\mathcal{H}): P T P=T P$ for all $P \in \mathcal{N}\}$.

Some other examples of local algebras are those of the form alg $\mathcal{P}$, where $\mathcal{P}$ is a commutative and completely distributive lattice of projections ([12]) and the Fourier Binest algebra introduced by Katavolos and Power ([11]). This binest algebra is defined as the intersection of the Volterra nest algebra and the analytical nest algebra, realized as operator algebras on the Hilbert space $L^{2}(\mathbf{R})$, and is shown to equal the weak-* closure of the Hilbert-Schmidt bianalytic pseudo-differential operators.

We now turn to the definition of the Bourgain algebra of an operator algebra.

In [7], the Bourgain algebra of an operator algebra $\mathcal{A} \subseteq \mathcal{L}(\mathcal{H})$ is defined by

$$
\mathcal{A}_{b}=\left\{T \in \mathcal{L}(\mathcal{H}): \operatorname{dist}\left(T A_{n}, \mathcal{A}\right) \rightarrow 0 \text { whenever } A_{n} \rightarrow 0 \text { weak-* in } \mathcal{A}\right\}
$$

It is straightforward to see that $\mathcal{A}_{b}$ is a norm-closed subalgebra of $\mathcal{L}(\mathcal{H})$ which contains the algebra $\mathcal{A}$. In the non-commutative setting of operator algebras, this definition is noticeably "one-sided". In the following, $\mathcal{A}_{b}$ will be referred to as the right Bourgain algebra of $\mathcal{A}$.

Accordingly, the left Bourgain algebra of $\mathcal{A}$ is hereby defined by

$$
{ }_{b} \mathcal{A}=\left\{T \in \mathcal{L}(\mathcal{H}): \operatorname{dist}\left(A_{n} T, \mathcal{A}\right) \rightarrow 0 \text { whenever } A_{n} \rightarrow 0 \text { weak-* in } \mathcal{A}\right\}
$$


while the two-sided Bourgain algebra, or, simply, the Bourgain algebra of $\mathcal{A}$ is given by

$$
\mathcal{A}_{B}=\mathcal{A}_{b} \cap_{b} \mathcal{A} \text {. }
$$

Again, it is not hard to show that the left Bourgain algebra, and hence the two-sided Bourgain algebra as well, is a norm-closed subalgebra of $\mathcal{L}(\mathcal{H})$ containing the algebra $\mathcal{A}$.

As the sequence $\left\{A_{n}\right\}$ tends to 0 weak-* in $\mathcal{A}$ if, and only if, the sequence of adjoints $\left\{A_{n}^{*}\right\}$ tends to 0 weak-* in the algebra $\mathcal{A}^{*}=\left\{A^{*}: A \in \mathcal{A}\right\}$, elementary considerations show that

$$
{ }_{b} \mathcal{A}=\left(\left(\mathcal{A}^{*}\right)_{b}\right)^{*}
$$

The proof of Lemma 2 in [7] actually enables us to establish the following stronger result whose proof we give for the sake of completeness.

Lemma 1.1. If $\mathcal{A}$ and $\mathcal{B}$ are subalgebras of $\mathcal{L}(\mathcal{H})$ such that $(\mathcal{A} \cap \mathcal{K})$ is weak-* dense in $\mathcal{A}$ and $\mathcal{A} \subseteq \mathcal{B}$, then $\mathcal{B}_{b}$ is contained in the norm closure of $\mathcal{B}+\mathcal{K}$. The same conclusion holds for the left and two-sided Bourgain algebras of $\mathcal{B}$.

Proof. If $\left\{R_{n}\right\}$ is a sequence in $\mathcal{A} \cap \mathcal{K}$ such that $\left(1-R_{n}\right) \rightarrow 0$ in the weak-* topology, then, as $\mathcal{A} \subseteq \mathcal{B}$, it follows that $\left(1-R_{n}\right) \rightarrow 0$ weak-* in $\mathcal{B}$ as well. For $T \notin \overline{\mathcal{B}+\mathcal{K}}$, we have

$$
\begin{aligned}
\operatorname{dist}\left(T\left(1-R_{n}\right), \mathcal{B}\right) & \geq \operatorname{dist}\left(T-T R_{n}, \overline{\mathcal{B}+\mathcal{K}}\right) \\
& =\operatorname{dist}(T, \overline{\mathcal{B}+\mathcal{K}})>0
\end{aligned}
$$

for each $n$. Hence, $T \notin \mathcal{B}_{b}$ which gives the result.

Only a small modification of the above argument is needed to establish the desired result for ${ }_{b} \mathcal{B}$ and, hence, for $\mathcal{B}_{B}$ as well.

If $\mathcal{A}$ is local, then applying the Lemma with $\mathcal{B}=\mathcal{A}$ shows that the (right, left, or two-sided) Bourgain algebra of $\mathcal{A}$ is contained in $\mathcal{A}+\mathcal{K}$, while taking $\mathcal{B}=\mathcal{A}+\mathcal{K}$ shows that $\mathcal{A}+\mathcal{K}=(\mathcal{A}+\mathcal{K})_{b}={ }_{b}(\mathcal{A}+\mathcal{K})=(\mathcal{A}+\mathcal{K})_{B}$.

More generally, if $\mathcal{A}$ is local and $\mathcal{A} \subseteq \mathcal{B} \subseteq \mathcal{A}+\mathcal{K}$, then $\overline{\mathcal{B}+\mathcal{K}} \subseteq \mathcal{A}+\mathcal{K}$ and it follows from the Lemma that the Bourgain algebra of $\mathcal{B}$ is contained in $\mathcal{A}+\mathcal{K}$.

\section{The nest algebra case}

For nest algebras, it was shown in [7] that both inclusions $\operatorname{alg} \mathcal{N} \subset \operatorname{alg} \mathcal{N}_{b}$ and $\operatorname{alg} \mathcal{N}_{b} \subset \operatorname{alg} \mathcal{N}+\mathcal{K}$ can be proper and that both equalities alg $\mathcal{N}=\operatorname{alg} \mathcal{N}_{b}$ and $\operatorname{alg} \mathcal{N}_{b}=\operatorname{alg} \mathcal{N}+\mathcal{K}$ can be attained by suitable choice of the nest. In Theorem 2.4 below, we give a complete characterization, in algebraic terms, of the right, left, and 
two-sided Bourgain algebras of a nest algebra. This characterization will, as it should do, illuminate the earlier examples.

We first require a bit more preliminary information and terminology. For $\mathcal{N}$ a nest and $P$ a projection in $\mathcal{N}$, define the projections $P_{-}=\sup \{E \in \mathcal{N}: E<P\}$ and $P_{+}=\inf \{E \in \mathcal{N}: E>P\}$. As the nest is complete (closed under suprema and infima), it follows that $P_{-}$and $P_{+}$are both in $\mathcal{N}$. An interval in $\mathcal{N}$ is a projection of the form $(F-E)$, where $E$ and $F$ are in $\mathcal{N}$ and $E<F$. A minimal interval is called an atom while the term proper interval will refer to an interval $(F-E)$, where $0<E<F<1$.

We will also make use of Arveson's well-known distance formula for nest algebras ([1]). Namely, for $\mathcal{N}$ a nest and $T$ in $\mathcal{L}(\mathcal{H})$,

$$
\operatorname{dist}(T, \operatorname{alg} \mathcal{N})=\sup \left\{\left\|P^{\perp} T P\right\|: P \in \mathcal{N}\right\} .
$$

Lastly, we need a basic result in the theory of nest algebras, due to Erdos ([5]), that the rank-one operator $x \otimes y^{*}$ belongs to alg $\mathcal{N}$ if, and only if, there is a projection $P$ in $\mathcal{N}$ such that $P x=x$ and $\left(P_{-}\right)^{\perp} y=y$. We are now ready to proceed.

Theorem 2.1. Let $\mathcal{N}$ be a nest and $\mathcal{A}=\operatorname{alg} \mathcal{N}$. If the compact operator $K$ belongs to $\mathcal{A}_{b}$, then $P_{-}<P<P_{+}$and $\left(P-P_{-}\right)$is finite-dimensional whenever $P^{\perp} K P \neq 0$ for $P \in \mathcal{N}$.

Proof. The result is clearly true if $K \in \mathcal{A}$, so assume that $K \in \mathcal{A}_{b} \backslash \mathcal{A}$ and let $P$ be a projection in the nest for which $R=P^{\perp} K P \neq 0$. Note that $R=P^{\perp} R P$ and that $R$ is compact. Also, as $\mathcal{A}_{b}$ is a norm-closed algebra containing $\mathcal{A}$ and $\mathcal{K}$, it follows that $\mathcal{A}_{b}$ contains the norm-closed algebra generated by $\mathcal{A}$ and $\mathcal{K}$. This includes the operator $R(=K P-P K P)$ so that $R \in \mathcal{A}_{b} \backslash \mathcal{A}$.

Suppose that $P_{+}=P$ and take a strictly decreasing sequence $\left\{Q_{n}\right\} \subseteq \mathcal{N}$ such that $Q_{n} \rightarrow P$ in the strong operator topology. Choose a unit vector $x_{0}$ such that $P x_{0}=x_{0}$ and $R x_{0} \neq 0$. Thus, $\left\|Q_{n}^{\perp} R x_{0}-R x_{0}\right\|=\left\|\left(Q_{n}^{\perp}-P^{\perp}\right) R x_{0}\right\| \rightarrow 0$, whence, there exists $n_{0}$ such that $\left\|Q_{n}^{\perp} R x_{0}\right\| \geq\left\|R x_{0}\right\| / 2$ for all $n \geq n_{0}$. For each $n \geq n_{0}$, let $y_{n}$ be a unit vector satisfying $\left(Q_{n}-Q_{n+1}\right) y_{n}=y_{n}$ and set $A_{n}=x_{0} \otimes y_{n}^{*}$.

Clearly, each $A_{n}$ belongs to $\mathcal{A}$ and, as $\left\{y_{n}\right\}$ is an orthonormal set, we see that the sequence $\left\{A_{n}\right\}$ tends to 0 in the weak-* topology.

However, for each $n \geq n_{0}$, we have

$$
\begin{aligned}
\operatorname{dist}\left(R A_{n}, \operatorname{alg} \mathcal{N}\right) & \geq\left\|Q_{n}^{\perp} R A_{n} Q_{n}\right\|=\left\|Q_{n}^{\perp} R x_{0} \otimes y_{n}^{*}\right\| \\
& =\left\|Q_{n}^{\perp} R x_{0}\right\| \geq\left\|R x_{0}\right\| / 2>0 .
\end{aligned}
$$

This contradicts the fact that $R \in \mathcal{A}_{b}$. We conclude that $P_{+}>P$.

Next, suppose that $P_{-}=P$ and this time take $\left\{Q_{n}\right\}$ to be a strictly increasing sequence of projections in $\mathcal{N}$ such that $Q_{n} \rightarrow P$ in the strong operator topology. Take $y$ to be unit vector such that $y=P y$ and $R y \neq 0$. The fact that $\left\|R y-R Q_{n} y\right\|=$ $\left\|R\left(P-Q_{n}\right) y\right\| \leq\|R\|\left\|\left(P-Q_{n}\right) y\right\| \rightarrow 0$ implies that $R Q_{m} y \neq 0$ for some $m$. Let $y_{0}=Q_{m} y$ 
and define $A_{n}=y_{0} \otimes x_{n}^{*}$ for each $n \geq m+1$, where $x_{n}$ is a unit vector satisfying $x_{n}=\left(Q_{n}-Q_{n-1}\right) x_{n}$. The usual calculations show that the sequence $\left\{A_{n}: n \geq m+1\right\}$ tends to 0 in the weak-* topology on $\mathcal{A}$. Now, for each $n \geq m+1$, we have

$$
\begin{aligned}
\operatorname{dist}\left(R A_{n}, \operatorname{alg} \mathcal{N}\right) & \geq\left\|P^{\perp} R A_{n} P\right\|=\left\|R y_{0} \otimes x_{n}^{*}\right\| \\
& =\left\|R y_{0}\right\|>0 .
\end{aligned}
$$

This contradicts the fact that $R \in \mathcal{A}_{b}$. We conclude that $P_{-}<P$.

Finally, suppose that the projection $\left(P-P_{-}\right)$is infinite-dimensional and take $\left\{x_{n}: n \geq 1\right\}$ to be an orthonormal set with $x_{n}=\left(P-P_{-}\right) x_{n}$ for every $n$. Choose $y_{0}$ such that $P y_{0}=y_{0}$ and $R y_{0} \neq 0$ and set $A_{n}=y_{0} \otimes x_{n}^{*}$. The sequence $\left\{A_{n}\right\}$ tends to 0 in the weak-* topology on $\mathcal{A}$, but, again contradicting the fact that $R \in \mathcal{A}_{b}$, we have, for each $n$,

$$
\begin{aligned}
\operatorname{dist}\left(R A_{n}, \operatorname{alg} \mathcal{N}\right) & \geq\left\|P^{\perp} R A_{n} P\right\|=\left\|R y_{0} \otimes x_{n}^{*}\right\| \\
& =\left\|R y_{0}\right\|>0
\end{aligned}
$$

It follows that $P_{-}<P<P_{+}$and that $\left(P-P_{-}\right)$is finite-dimensional, thus completing the proof of the theorem.

Corollary 2.2. For a nest algebra $\mathcal{A}=\operatorname{alg} \mathcal{N}$, we have

(i) if $K \in{ }_{b} \mathcal{A} \cap \mathcal{K}$, then $P_{-}<P<P_{+}$and $\left(P_{+}-P\right)$ is finite-dimensional whenever $P^{\perp} K P \neq 0$ for $P \in \mathcal{N}$, and

(ii) if $K \in \mathcal{A}_{B} \cap \mathcal{K}$, then $P_{-}<P<P_{+}$and $\left(P_{+}-P_{-}\right)$is finite-dimensional for all $P \in \mathcal{N}$ such that $P^{\perp} K P \neq 0$.

Proof. To prove (i), apply the Theorem to the nest algebra $\mathcal{A}^{*}=\operatorname{alg} \mathcal{N}^{\perp}$, where $\mathcal{N}^{\perp}=\left\{P^{\perp}: P \in \mathcal{N}\right\}$, using the relations $\left(P^{\perp}\right)_{-}=\left(P_{+}\right)^{\perp},\left(P^{\perp}\right)_{+}=\left(P_{-}\right)^{\perp}$, and the fact that $Q^{\perp}-P^{\perp}=P-Q$. Then use the relation ${ }_{b} \mathcal{A}=\left(\left(\mathcal{A}^{*}\right)_{b}\right)^{*}$.

For the second assertion, suppose that $K \in \mathcal{A}_{B} \cap \mathcal{K}$ and that $P^{\perp} K P \neq 0$ for some $P \in \mathcal{N}$. As $K \in \mathcal{A}_{b} \cap \mathcal{K}$, it follows from the Theorem that $P_{-}<P<P_{+}$and that $\left(P-P_{-}\right)$is finite-dimensional. At the same time, we have that $K \in_{b} \mathcal{A} \cap \mathcal{K}$, so it follows from the first part of this Corollary that $\left(P_{+}-P\right)$ is also finite-dimensional. Thus, $\left(P_{+}-P_{-}\right)$is finite-dimensional as desired.

Remark. Let $\mathcal{A}=\operatorname{alg} \mathcal{N}$ be a nest algebra. Example 2 of [7] shows that, if there is a projection $P$ in the nest for which $P_{-}<P<P_{+}$and $\left(P-P_{-}\right)$is finite-dimensional, then there exists a compact operator $K \in \mathcal{A}_{b} \backslash \mathcal{A}$ and, therefore, $\mathcal{A}$ is properly contained in $\mathcal{A}_{b}$. Together with the Theorem, this implies that, for a nest algebra $\mathcal{A}$, the equality $\mathcal{A}=\mathcal{A}_{b}$ holds if, and only if, the projection $\left(P-P_{-}\right)$is infinite-dimensional whenever $P_{-}<P<P_{+}$for $P$ in the nest. In particular, this applies if the nest is continuous.

A construction similar to that of Example 2 of [7] can be given which, together with 
part (i) of Corollary 2.2, shows that, for a nest algebra $\mathcal{A}=\operatorname{alg} \mathcal{N}$, the equality $\mathcal{A}={ }_{b} \mathcal{A}$ holds if, and only if, the projection $\left(P_{+}-P\right)$ is infinite-dimensional whenever $P_{-}<P<P_{+}$for $P \in \mathcal{N}$. Again, this applies to all continuous nests.

Of course, if either $\mathcal{A}_{b}=\mathcal{A}$ or ${ }_{b} \mathcal{A}=\mathcal{A}$, then we will have $\mathcal{A}_{B}=\mathcal{A}$. But, consideration of the above results as they apply to the example $\mathcal{N}=\{0, P, Q, 1\}$, where $P$ and $Q^{\perp}$ are finite-dimensional while $(Q-P)$ is infinite-dimensional, shows that, in this case, both the right and left Bourgain algebras of alg $\mathcal{N}$ properly contain alg $\mathcal{N}$ while their intersection, the two-sided Bourgain algebra, coincides with alg $\mathcal{N}$.

In order to establish the algebraic characterization of the Bourgain algebra of a nest algebra, we require the examples discussed in the following proposition.

Proposition 2.3. Let $\mathcal{N}$ be a non-trivial nest. Each of the following four conditions implies that $(\operatorname{alg} \mathcal{N})_{b}=\operatorname{alg} \mathcal{N}+\mathcal{K}$.

(i) $\mathcal{N}=\left\{P_{n}:-\infty<n<\infty\right\} \cup\{0,1\}$, where each atom $\left(P_{n+1}-P_{n}\right)$ is finitedimensional;

(ii) $\mathcal{N}=\left\{P_{n}: n \geq 1\right\} \cup\{0,1\}$, where each $P_{n}$ is finite-dimensional;

(iii) $\mathcal{N}=\left\{P_{n}:-\infty<n \leq-1\right\} \cup\{0,1\}$, where $\left(P_{n}-P_{n-1}\right)$ is finite-dimensional for each $n \leq-1$

(iv) $\mathcal{N}=\left\{P_{1}, \ldots, P_{M}\right\} \cup\{0,1\}$, for some natural number $M$, where $P_{k}$ is finitedimensional for each $k=1, \ldots, M$.

Proof. The first assertion is established in Example 1 of [7] but we give the argument again here for the sake of completeness. The proofs of the other cases mainly involve modifications of this first proof.

(i) Let $\mathcal{N}=\left\{P_{n}:-\infty<n<\infty\right\} \cup\{0,1\}$ and, for simplicity, assume that each atom $\left(P_{n}-P_{n-1}\right)$ is one-dimensional with range spanned by the unit vector $e_{n}$. Thus, the set $\left\{e_{n}:-\infty<n<\infty\right\}$ is an orthonormal basis for $\mathcal{H}$. As $(\operatorname{alg} \mathcal{N})_{b}$ is a norm-closed subalgebra of alg $\mathcal{N}+\mathcal{K}$, it suffices to show that each of the rank-one operators $e_{n} \otimes e_{m}^{*}$, for integers $m$ and $n$, belongs to (alg $\left.\mathcal{N}\right)_{b}$. For this, choose integers $m$ and $n$ and set $R=e_{n} \otimes e_{m}^{*}$. If $m \geq n$, then $R \in \operatorname{alg} \mathcal{N} \subseteq(\operatorname{alg} \mathcal{N})_{b}$ whilst, if $m<n$ and $T \in \operatorname{alg} \mathcal{N}$, then, using Arveson's distance formula, we have

$$
\operatorname{dist}(R T, \operatorname{alg} \mathcal{N})=\sup _{-\infty<j<\infty}\left\|P_{j}^{\perp} R T P_{j}\right\|=\left(\sum_{k=m}^{n-1}\left|\left(T e_{k}, e_{m}\right)\right|^{2}\right)^{1 / 2} .
$$

Finally, if the operators $\left\{T_{j}\right\}$ in alg $\mathcal{N}$ converge to 0 in the weak-* topology, then, for each $k$ with $m \leq k \leq n-1$, we have $\lim _{j \rightarrow \infty}\left|\left(T_{j} e_{k}, e_{m}\right)\right|=0$. As there are only finitely many such $k$ to consider, it follows that $\lim _{j \rightarrow \infty} \operatorname{dist}\left(R T_{j}, \operatorname{alg} \mathcal{N}\right)=0$ and, hence, that $R \in(\operatorname{alg} \mathcal{N})_{b}$ as desired.

The argument requires only slight modification if the atoms $\left(P_{n}-P_{n-1}\right)$ are assumed merely to be finite-dimensional. 
(ii) Consider now the case where $\mathcal{N}=\left\{P_{n}: n \geq 1\right\} \cup\{0,1\}$, where each $P_{k}$ is finitedimensional. Again, the argument is simplified if we assume that each atom $\left(P_{n}-P_{n-1}\right)$ (where we take $P_{0}=0$ ) is one-dimensional with range spanned by the unit vector $e_{n}$. Thus, $\left\{e_{n}: n \geq 1\right\}$ is an orthonormal basis for $\mathcal{H}$. As in the proof of the first assertion, let $R=e_{n} \otimes e_{m}^{*}$. If $m \geq n$ then $R \in \operatorname{alg} \mathcal{N}$ so assume that $m<n$. For $T \in \operatorname{alg} \mathcal{N}$ we then have

$$
\operatorname{dist}(R T, \operatorname{alg} \mathcal{N})=\left(\sum_{k=m}^{n-1}\left|\left(T e_{k}, e_{m}\right)\right|^{2}\right)^{1 / 2}
$$

as before. The rest of the proof that $R \in(\operatorname{alg} \mathcal{N})_{b}$ proceeds as above.

(iii) Let $\mathcal{N}=\left\{P_{n}:-\infty<n \leq-1\right\} \cup\{0,1\}$, where, for simplicity again, we assume that each atom $\left(P_{n}-P_{n-1}\right)$ is one-dimensional with range spanned by the unit vector $e_{n}$. Thus, $\left\{e_{n}: n \leq-1\right\}$ is an orthonormal basis for the range of $P_{-1}$. The previous arguments show that $e_{n} \otimes e_{m}^{*} \in(\operatorname{alg} \mathcal{N})_{b}$ for all negative integers $m$ and $n$.

Next, observe that, if $R=x \otimes y^{*}$, where $x$ is arbitrary and $y=\left(1-P_{-1}\right) y$, then $R P_{k}=0$ for all $k \leq-1$ so that $R \in \operatorname{alg} \mathcal{N} \subseteq(\operatorname{alg} \mathcal{N})_{b}$.

Finally, suppose that $R=x \otimes e_{m}^{*}$, where $x=\left(1-P_{-1}\right) x$ and $m \leq-1$. Then, for $T \in \operatorname{alg} \mathcal{N}$, we have

$$
\operatorname{dist}(R T, \operatorname{alg} \mathcal{N})=\left\|P_{-1} T^{*} e_{m}\right\|=\left(\sum_{k=m}^{-1}\left|\left(T e_{k}, e_{m}\right)\right|^{2}\right)^{1 / 2}
$$

As before, if the sequence $\left\{T_{j}\right\}$ converges to 0 weak-* in $\operatorname{alg} \mathcal{N}$, then it follows that $\operatorname{dist}\left(R T_{j}, \operatorname{alg} \mathcal{N}\right) \rightarrow 0$ so that $R \in(\operatorname{alg} \mathcal{N})_{b}$. Again, as $(\operatorname{alg} \mathcal{N})_{b}$ is a norm-closed algebra, we conclude that $(\operatorname{alg} \mathcal{N})_{b}$ contains $\mathcal{K}$ and, hence, that the desired equality holds.

(iv) The argument is a simple modification of the ones just given above. This completes the proof.

In what follows, let $\mathcal{N}$ be a nest of projections with $\mathcal{A}=\operatorname{alg} \mathcal{N}$. Define three additional nests, $\mathcal{N}_{r}, \mathcal{N}_{l}$, and $\mathcal{N}_{\infty}$, like so.

$$
\begin{aligned}
\mathcal{N}_{r} & =\mathcal{N} \backslash\left\{P \in \mathcal{N}: P_{-}<P<P_{+} \text {and } \operatorname{dim}\left(P-P_{-}\right)<\infty\right\} \\
\mathcal{N}_{1} & =\mathcal{N} \backslash\left\{P \in \mathcal{N}: P_{-}<P<P_{+} \text {and } \operatorname{dim}\left(P_{+}-P\right)<\infty\right\} \\
\mathcal{N}_{\infty} & =\mathcal{N} \backslash\left\{P \in \mathcal{N}: P_{-}<P<P_{+} \text {and } \operatorname{dim}\left(P_{+}-P_{-}\right)<\infty\right\}
\end{aligned}
$$

Observe that each of these is a complete nest as no strong limit $P$ of $\mathcal{N}$ can satisfy $P_{-}<P<P_{+}$. Let $\mathcal{A}_{r}, \mathcal{A}_{1}$, and $\mathcal{A}_{\infty}$ be the corresponding nest algebras. A moment's reflection shows that $\mathcal{N}_{r} \cup \mathcal{N}_{l}=\mathcal{N}_{\infty} \subseteq \mathcal{N}$ and, therefore, that $\mathcal{A}_{r} \cap \mathcal{A}_{l}=\mathcal{A}_{\infty} \supseteq \mathcal{A}$.

We now give our main result. 
Theorem 2.4. Let $\mathcal{A}, \mathcal{A}_{r}, \mathcal{A}_{l}$ and $\mathcal{A}_{\infty}$ be as in the preceding paragraph. Then

(i) $\mathcal{A}_{b}=(\mathcal{A}+\mathcal{K}) \cap \mathcal{A}_{\text {, }}=\mathcal{A}+\left(\mathcal{A}_{\text {r }} \cap \mathcal{K}\right)$,

(ii) ${ }_{b} \mathcal{A}=(\mathcal{A}+\mathcal{K}) \cap \mathcal{A}_{l}=\mathcal{A}+\left(\mathcal{A}_{l}+\mathcal{K}\right)$, and

(iii) $\mathcal{A}_{B}=(\mathcal{A}+\mathcal{K}) \cap \mathcal{A}_{\infty}=\mathcal{A}+\left(\mathcal{A}_{\infty} \cap \mathcal{K}\right)$.

Proof. It is straightforward to show that $(\mathcal{A}+\mathcal{K}) \cap \mathcal{B}=\mathcal{A}+(\mathcal{B} \cap \mathcal{K})$ for any subalgebra $\mathcal{B}$ of $\mathcal{L}(\mathcal{H})$ which contains $\mathcal{A}$. To establish the first assertion, let $T \in \mathcal{A}_{b} \subseteq \mathcal{A}+\mathcal{K}$ and write $T=A+K$ with $A \in \mathcal{A}$ and $K \in \mathcal{K}$. As $\mathcal{A} \subseteq \mathcal{A}_{b}$, it follows that $K \in \mathcal{A}_{b}$. Theorem 2.1 then implies that $P^{\perp} K P=0$ for all $P \in \mathcal{N}_{r}$; that is, $K \in \mathcal{A}_{r}$. This shows that $\mathcal{A}_{b} \subseteq \mathcal{A}+\left(\mathcal{A}_{r} \cap \mathcal{K}\right)$.

For the reverse inclusion, observe that, as $\mathcal{A} \subseteq \mathcal{A}_{b}$ and $\mathcal{A}_{b}$ is an algebra, it suffices to show that $\left(\mathcal{A}_{r} \cap \mathcal{K}\right) \subseteq \mathcal{A}_{b}$. For this, let $K \in \mathcal{A}_{r} \cap \mathcal{K}$ and take $A \in \mathcal{A}$. As $K A \in \mathcal{A}_{r}$, we have $E^{\perp} K E=0$ for all $E \in \mathcal{N}_{r}$. Thus,

$$
\operatorname{dist}(K A, \mathcal{A})=\sup \left\{\left\|E^{\perp} K A E\right\|: E \in \mathcal{N}_{0}\right\}
$$

where $\mathcal{N}_{0}=\left\{P \in \mathcal{N}: P_{-}<P<P_{+}\right.$and $\left.\operatorname{dim}\left(P-P_{-}\right)<\infty\right\}$.

Now, for each $E \in \overline{\mathcal{N}}_{0}$, there exist projections $P$ and $Q$ in $\mathcal{N}_{r}$ such that $P<E<Q$ and $(Q-P)$ is an atom of the nest $\mathcal{N}_{r}$. (Take $P=\sup \left\{F \in \mathcal{N}_{r}: F<E\right\}$ and $Q=\inf \left\{F \in \mathcal{N}_{r}: F>E\right\}$. Then no $F$ in $\mathcal{N}_{r}$ can satisfy $P<F<Q$.) For such $E, P$ and $Q$, we have $P^{\perp} K A P=Q^{\perp} K A Q=0$ and, thus, $E^{\perp} K A E=E^{\perp}(Q-P) K A(Q-P) E$. Moreover, $(Q-P) K=(Q-P) K P^{\perp}$ and $A(Q-P)=Q A(Q-P)$ so that

$$
\begin{aligned}
E^{\perp} K A E & =E^{\perp}[(Q-P) K(Q-P)][(Q-P) A(Q-P)] E \\
& =\left[(Q-P) E^{\perp}\right][(Q-P) K(Q-P)][(Q-P) A(Q-P)][(Q-P) E] .
\end{aligned}
$$

Consider now the nest $\hat{\mathcal{N}}=(Q-P) \mathcal{N}$ with corresponding nest algebra $\hat{\mathcal{A}}$ defined on the Hilbert space $(Q-P) \mathcal{H}$. Because there are no strong limit points of $\mathcal{N}$ strictly between $P$ and $Q$ and no infinite-dimensional proper subintervals of $(Q-P)$, the nest $\hat{\mathcal{N}}$ is either trivial or it satisfies one of the four conditions in the statement of Proposition 2.3. In the former case, we have $Q=P_{+}$in $\mathcal{N}$ so that $(Q-P) K(Q-P) \in \hat{\mathcal{A}} \subseteq \hat{\mathcal{A}}_{b}$ while, in the latter case, we have, from Proposition 2.3, that $(\hat{\mathcal{A}})_{b}=\hat{\mathcal{A}}+(Q-P) \mathcal{K}(Q-P)$ which again implies that $(Q-P) K(Q-P) \in \hat{\mathcal{A}}_{b}$. In either case, therefore, if $A_{n} \rightarrow 0$ weak-* in $\mathcal{A}$, then $(Q-P) A_{n}(Q-P) \rightarrow 0$ weak-* in $\hat{\mathcal{A}}$ and $\lim _{n \rightarrow \infty} \sup \left\{\left\|E^{\perp} K A_{n} E\right\|: E \in \mathcal{N}_{0}, P<E<Q\right\}=0$.

We have just seen that, if $K \in \mathcal{A}_{r} \cap \mathcal{K},(Q-P)$ is an atom in $\mathcal{N}_{r}$, and $\left\{A_{n}\right\}$ is a weak-* null sequence in $\mathcal{A}$, then $\lim _{n \rightarrow \infty} \sup \left\{\left\|E^{\perp} K A_{n} E\right\|: E \in \mathcal{N}_{0}, P<E<Q\right\}=0$. If there are only finitely many such atoms, say $\left(Q_{1}-P_{1}\right), \ldots,\left(Q_{m}-P_{m}\right)$, then, given $\epsilon>0$, we may choose, for each $k=1, \ldots, m$, a natural number $n_{k}$ such that $\sup \left\{\left\|E^{\perp} K A_{n} E\right\|: E \in \mathcal{N}_{0}, P_{k}<E<Q_{k}\right\}<\epsilon$ for all $n \geq n_{k}$. With $N=\max \left\{n_{1}, \ldots, n_{m}\right\}$, we have $\left\|E^{\perp} K A_{n} E\right\|<\epsilon$ for all $n \geq N$ and all $E \in \mathcal{N}_{0}$ from which it follows that $\operatorname{dist}\left(K A_{n}, \mathcal{A}\right)<\epsilon$ for all $n \geq N$. Therefore, $K \in \mathcal{A}_{b}$ as desired. 
Suppose, instead, that there are infinitely many such atoms, say $\left\{\left(Q_{i}-P_{i}\right): i \in \mathcal{Z}\right\}$. As the sequence $\left\{A_{n}\right\}$ is weak-* null, the set of norms $\left\{\left\|A_{n}\right\|\right\}$ is bounded, say by $M$. Because $K$ is compact, there exists, for a given $\epsilon>0$, a natural number $m$ such that $\left\|\left(Q_{i}-P_{i}\right) K\right\|<\epsilon / M$ whenever $|i| \geq m$. Thus, if $|i| \geq m$ and $P_{i}<E<Q_{i}$ for $E \in \mathcal{N}_{0}$, then $\left\|E^{\perp} K A_{n} E\right\| \leq\left\|\left(Q_{i}-P_{i}\right) K A_{n}\left(Q_{i}-P_{i}\right)\right\|<(\epsilon / M) \cdot M=\epsilon$. Next, as in the previous paragraph, we may choose a natural number $N$ such that $\sup \left\{\left\|E^{\perp} K A_{n} E\right\|: E \in \mathcal{N}_{0}, P_{i}<E<Q_{i}\right\}<\epsilon$ for all $n \geq N$ and all $i$ with $|i| \leq m$. We thus have that $\left\|E^{\perp} K A_{n} E\right\|<\epsilon$ for all $E \in \mathcal{N}_{0}$ and all $n \geq N$. Hence, $\lim _{n \rightarrow \infty} \operatorname{dist}\left(K A_{n}, \mathcal{A}\right)=0$ so that $K \in \mathcal{A}_{b}$ as desired. This completes the proof that $\mathcal{A}_{r} \cap \mathcal{K} \subseteq \mathcal{A}_{b}$ and the first assertion of the Theorem now follows.

To prove the second assertion requires only the obvious modifications of the arguments just given while the third assertion follows immediately from the first two as soon as one recalls that $\mathcal{A}_{B}=\mathcal{A}_{b} \cap{ }_{b} \mathcal{A}$ and that $\mathcal{A}_{\infty}=\mathcal{A}_{r} \cap \mathcal{A}_{l}$. The proof of the Theorem is now complete.

Corollary 2.5. Let $\mathcal{A}=\operatorname{alg} \mathcal{N}$ be a nest algebra. Then

(i) $\mathcal{A}_{b}=\mathcal{A}$ if, and only if, the projection $\left(P-P_{-}\right)$is infinite-dimensional whenever $P_{-}<P<P_{+}$for $P \in \mathcal{N}$.

(ii) ${ }_{b} \mathcal{A}=\mathcal{A}$ if, and only if, the projection $\left(P_{+}-P\right)$ is infinite-dimensional whenever $P_{-}<P<P_{+}$for $P \in \mathcal{N}$, and

(iii) $\mathcal{A}_{B}=\mathcal{A}$ if, and only if, the projection $\left(P_{+}-P_{-}\right)$is infinite-dimensional whenever $P_{-}<P<P_{+}$for $P \in \mathcal{N}$.

Proof. The Theorem implies that the stated equalities occur if, and only if, $\mathcal{A}_{r}=\mathcal{A}, \mathcal{A}_{t}=\mathcal{A}$, and $\mathcal{A}_{\infty}=\mathcal{A}$, respectively. These, in turn, occur if, and only if, $\mathcal{N}_{r}=\mathcal{N}, \mathcal{N}_{l}=\mathcal{N}$, and $\mathcal{N}_{\infty}=\mathcal{N}$, respectively. The Corollary now follows.

An alternative proof of the first two assertions of Corollary 2.5 was discussed in the remarks following Corollary 2.2. Similarly, for the third assertion, it follows from Theorem 2.1 that the condition that $\left(P_{+}-P_{-}\right)$is infinite-dimensional whenever $P_{-}<P<P_{+}$for $P \in \mathcal{N}$ implies that $\mathcal{A}_{B}=\mathcal{A}$. Conversely, if there is a projection $P$ in $\mathcal{N}$ such that $P_{-}<P<P_{+}$and $\left(P_{+}-P_{-}\right)$is finite-dimensional, then one can show directly that any operator $R$ satisfying $R=\left(P_{+}-P\right) R\left(P-P_{-}\right)$belongs to $\mathcal{A}_{B}$ and, hence, that $\mathcal{A}_{B} \neq \mathcal{A}$.

Corollary 2.6. Let $\mathcal{N}$ be a non-trivial nest of projections and $\mathcal{A}=\operatorname{alg} \mathcal{N}$. The following statements hold.

(i) $\mathcal{A}_{b}=\mathcal{A}+\mathcal{K}$ if, and only if, all proper intervals in $\mathcal{N}$ as well as the projection $0_{+} \equiv \inf \{E \in \mathcal{N}: E>0\}$ are finite-dimensional;

(ii) ${ }_{b} \mathcal{A}=\mathcal{A}+\mathcal{K}$ if, and only if, all proper intervals in $\mathcal{N}$ as well as the projection $\left(1_{-}\right)^{\perp}$ are finite-dimensional; 
(iii) $\mathcal{A}_{B}=\mathcal{A}+\mathcal{K}$ if, and only if, every proper interval in $\mathcal{N}$ is finite-dimensional and both $0_{+}$and $\left(1_{-}\right)^{\perp}$ are finite-dimensional.

Proof. To establish the first assertion, observe that, if all proper intervals in $\mathcal{N}$ as well as the projection $0_{+} \equiv \inf \{E \in \mathcal{N}: E>0\}$ are finite-dimensional, then $\mathcal{N}_{r}=\{0,1\}$ so that $\mathcal{A},=\mathcal{L}(\mathcal{H})$. The Theorem then implies that $\mathcal{A}_{b}=\mathcal{A}+\mathcal{K} \cap \mathcal{L}(\mathcal{H})=\mathcal{A}+\mathcal{K}$. Conversely, if $\mathcal{A}_{b}=\mathcal{A}+\mathcal{K}$, then, by the Theorem, we must have $\mathcal{N}_{r}=\{0,1\}$. This implies that $P_{-}<P<P_{+}$and that $\left(P-P_{-}\right)$is finite-dimensional for all non-trivial $P$ in $\mathcal{N}$. Consequently, either $0_{+}=0$ or $0<0_{+}<1$ (here we use the assumption that the nest is non-trivial) in which case $0=\left(0_{+}\right)_{-}$. In either case, $0_{+}$is finite-dimensional. Moreover, as all strong limits of $\mathcal{N}$ are in $\mathcal{N}_{r}$, it follows that, if $(F-E)$ is a proper interval in $\mathcal{N}$, then there can be only finitely many elements of $\mathcal{N}$ between $E$ and $F$. That is, we have $0<E=E_{1}<\ldots<E_{m}=F<1$ where each interval $\left(E_{k+1}-E_{k}\right)$ for $k=1, \ldots, m-1$ is an atom in $\mathcal{N}$. The hypotheses imply that $\left(E_{k+1}-E_{k}\right)$ is finitedimensional for each $k=1, \ldots, m-1$ from which it follows that $(F-E)$ is finitedimensional as well.

The second assertion follows from the usual modifications of the above arguments while the third statement follows from the first two together with the fact, obvious from the definition and Lemma 1.1, that $\mathcal{A}_{B}=\mathcal{A}+\mathcal{K}$ if, and only if, $\mathcal{A}_{b}={ }_{b} \mathcal{A}=\mathcal{A}+\mathcal{K}$.

As an alternative, one can prove Corollary 2.6 by showing that the condition that all proper intervals in $\mathcal{N}$ together with $0_{+}$are finite-dimensional implies that $\mathcal{N}$ has one of the four forms given in Proposition 2.3 (according to whether or not 0 and 1 are strong limits in $\mathcal{N}$ ). This implies that $\mathcal{A}_{b}=\mathcal{A}+\mathcal{K}$. Then use Example 3 of [7] to show that, if the nest contains projections $P$ and $Q$ with $0<P<Q<1$ and with $(Q-P)$ of infinite rank, then $\mathcal{A}_{b}$ is properly contained in $\mathcal{A}+\mathcal{K}$. A similar construction shows that $\mathcal{A}+\mathcal{K}$ will properly contain $\mathcal{A}_{b}$ if $0_{+}$is infinite-dimensional. Thus, the equality $\mathcal{A}_{b}=\mathcal{A}+\mathcal{K}$ holds precisely when the nest is of one of the four types given in Proposition 2.3.

Along the same lines, we see that the equality $\mathcal{A}_{B}=\mathcal{A}+\mathcal{K}$ occurs exactly when the nest $\mathcal{N}$ is of one of the following types.

(i) $\mathcal{N}=\left\{P_{n}: n \in \mathcal{Z}\right\} \cup\{0,1\}$, with $\operatorname{dim}\left(P_{n+1}-P_{n}\right)<\infty$ for all $n$ :

(ii) $\mathcal{N}=\left\{P_{n}: n \geq 1\right\} \cup\{0,1\}$, with each $P_{n}$ finite-dimensional;

(iii) $\mathcal{N}=\left\{P_{n}: n \leq-1\right\} \cup\{0,1\}$, with $\operatorname{dim}\left(P_{n}-P_{n-1}\right)<\infty$ for all $n$ and $\operatorname{dim}\left(1-P_{-1}\right)<\infty$

(iv) $\mathcal{N}=\left\{P_{1}, \ldots, P_{M}\right\} \cup\{0,1\}$, for some natural number $M$, with each $P_{k}$ finitedimensional and $\left(P_{M}\right)^{\perp}$ finite-dimensional (so that alg $\mathcal{N}$ is a finite-dimensional algebra of block upper-triangular matrices). 


\section{Higher order Bourgain algebras}

For a subalgebra $\mathcal{A}$ of $\mathcal{L}(\mathcal{H})$, we can define higher order (two-sided) Bourgain algebras of $\mathcal{A}$ by

$$
(\mathcal{A})_{B^{(n)}} \equiv\left(\left(\mathcal{A}_{B}\right) \ldots\right)_{B},(n \text { times })
$$

for $n \geq 1$. Similarly we can define the $n$th order right and left Bourgain algebras, $(\mathcal{A})_{b^{(n)}}$ and $b^{(n)}(\mathcal{A})$, respectively. Higher order "mixed" Bourgain algebras can be defined as well. In general, as each of these is again a norm-closed subalgebra of $\mathcal{L}(\mathcal{H})$, we have that every $(n+1)$-st order (two-sided, right, left, or mixed) Bourgain algebra of $\mathcal{A}$ contains every $n$th order (two-sided, right, left, or mixed) Bourgain algebra of $\mathcal{A}$ and each of these contains $\mathcal{A}$ itself.

In the case that the algebra $\mathcal{A}$ is local, that is, $\mathcal{A} \cap \mathcal{K}$ is weak-* dense in $\mathcal{A}$, then more can be said. Indeed, for any subalgebra $\mathcal{B}$ of $\mathcal{L}(\mathcal{H})$ satisfying $\mathcal{A} \subseteq \mathcal{B}$, it is then an immediate consequence of Lemma 1.1 that every higher order (two-sided, right, left, or mixed) Bourgain algebra of $\mathcal{B}$ is contained in $\overline{\mathcal{B}+\mathcal{K}}$. If, in addition, $\mathcal{B} \subseteq \mathcal{A}+\mathcal{K}$, then every higher order Bourgain algebra of $\mathcal{B}$ is contained in $\mathcal{A}+\mathcal{K}$.

Returning now to the nest algebra case, let $\mathcal{N}$ be a nest and $\mathcal{A}=\operatorname{alg} \mathcal{N}$ the corresponding nest algebra. Let $\mathcal{N}_{r}, \mathcal{N}_{l}, \mathcal{N}_{\infty}, \mathcal{A}_{r}, \mathcal{A}_{l}$, and $\mathcal{A}_{\infty}$ be as defined in the previous section.

It is readily apparent that $\left(\mathcal{N}_{\infty}\right)_{\infty}=\mathcal{N}_{\infty}$. Indeed, no projection $P$ in $\mathcal{N}_{\infty}$ can satisfy the conditions $P_{-}<P<P_{+}$and $\operatorname{dim}\left(P_{+}-P_{-}\right)<\infty$ within the nest $\mathcal{N}_{\infty}$ as these conditions would then have to have been satisfied by $P$ within the nest $\mathcal{N}$ itself.

The situation is not quite so tidy for the nests $\left(\mathcal{N}_{\mathrm{r}}\right)_{\text {, }}$ and $\left(\mathcal{N}_{l}\right)_{1}$. For example, if $\mathcal{N}=\{0, Q, 1\} \cup\left\{P_{n}: n \geq 1\right\}$, where $Q$ is finite-rank and where the projections $P_{n}$ are decreasing strongly to $Q$ with finite-dimensional atoms $\left(P_{n}-P_{n+1}\right)$, then $Q \in \mathcal{N}_{r} \backslash\left(\mathcal{N}_{r}\right)$. However, the nest $\mathcal{N}_{r}$ cannot contain two adjacent finite-dimensional atoms $(P-E)$ and $(F-P)$ as this would imply both $P_{-}<P<P_{+}$and $\operatorname{dim}\left(P-P_{-}\right)<\infty$ in $\mathcal{N}$. From this it follows that $\left(\left(\mathcal{N}_{r}\right)\right)=\left(\mathcal{N}_{r}\right)_{r}$. To see this, suppose that $(P-E)$ and $(F-P)$ are adjacent atoms in $\left(\mathcal{N}_{r}\right)$, with $\operatorname{dim}(P-E)<\infty$. As $\mathcal{N}_{r}$ cannot contain adjacent finitedimensional atoms, we have that $E=P_{-}$in the nest $\mathcal{N}_{r}$. But $P \in\left(\mathcal{N}_{r}\right)$, so we must have $P=P_{+}$in $\mathcal{N}_{r}$. This implies that the gap in $\mathcal{N}_{r}$ between $F$ and $P$ fits either description (i) or (iii) of Proposition 2.3. This, however, implies the existence of infinitely many pairs of adjacent finite-dimensional atoms in $\mathcal{N}_{r}$, an impossibility. We conclude that such a projection $P$ cannot exist in $\left(\mathcal{N}_{r}\right)_{r}$; that is, $\left(\left(\mathcal{N}_{r}\right)_{r}\right)=\left(\mathcal{N}_{r}\right)_{r}$. Similar statements apply to the nests $\left(\mathcal{N}_{l}\right)_{1}$ and $\left(\left(\mathcal{N}_{l}\right)_{1}\right)_{i}$.

We are now able to characterize all nine possible second-order (right, left, two-sided, and mixed) Bourgain algebras of a nest algebra.

Theorem 3.1. Let $\mathcal{N}$ be a nest with corresponding nest algebra $\mathcal{A}=\operatorname{alg} \mathcal{N}$. Then the following statements hold. 
(i) $\left(\mathcal{A}_{B}\right)_{b}=(\mathcal{A}+\mathcal{K}) \cap\left(\mathcal{A}_{\infty}\right)_{r}$;

(ii) ${ }_{b}\left(\mathcal{A}_{B}\right)=(\mathcal{A}+\mathcal{K}) \cap\left(\mathcal{A}_{\infty}\right)_{1}$;

(iii) $\left(\mathcal{A}_{B}\right)_{B}=(\mathcal{A}+\mathcal{K}) \cap\left(\mathcal{A}_{\infty}\right)_{\infty}$;

(iv) $\left(\mathcal{A}_{b}\right)_{b}=(\mathcal{A}+\mathcal{K}) \cap\left(\mathcal{A}_{r}\right)_{r}$;

(v) ${ }_{b}\left(\mathcal{A}_{b}\right)=(\mathcal{A}+\mathcal{K}) \cap\left(\mathcal{A}_{\tau}\right)_{i}$;

(vi) $\left(\mathcal{A}_{b}\right)_{B}=(\mathcal{A}+\mathcal{K}) \cap\left(\mathcal{A}_{r}\right)_{\infty}$;

(vii) ${ }_{b}\left({ }_{b} \mathcal{A}\right)=(\mathcal{A}+\mathcal{K}) \cap\left(\mathcal{A}_{l}\right)$;

(viii) $\left({ }_{b} \mathcal{A}\right)_{b}=(\mathcal{A}+\mathcal{K}) \cap\left(\mathcal{A}_{l}\right)_{r}$;

(ix) $\left({ }_{b} \mathcal{A}\right)_{B}=(\mathcal{A}+\mathcal{K}) \cap\left(\mathcal{A}_{l}\right)_{\infty}$.

Proof. First observe that each of the statements (iii), (vi) and (ix) follows from the two preceding it. The proofs of the other statements are all similar so we herewith give the proof of (iv) as being typical. As usual, statements involving left-sided Bourgain algebras are established from the right-sided case by switching to adjoints and using the relation $\left({ }_{b} \mathcal{A}\right)^{*}=\left(\mathcal{A}^{*}\right)_{b}$.

To establish the inclusion $\left(\mathcal{A}_{b}\right)_{b} \subseteq(\mathcal{A}+\mathcal{K}) \cap\left(\mathcal{A}_{r}\right)_{r}$, let $T \in\left(\mathcal{A}_{b}\right)_{b}$ be given and write $T=A+K$ with $A \in \mathcal{A}$ and $K \in \mathcal{K}$. If $P \in \mathcal{N}_{r}$ satisfies $P^{\perp} T P \neq 0$, then $P^{\perp} K P \neq 0$ as well and, setting $R=P^{\perp} K P$, we have that $R \in\left(\mathcal{A}_{b}\right)_{b} \cap \mathcal{K}$. Now follow the proof of Theorem 2.1 to the letter, but working in $\mathcal{N}_{r}$ instead of in $\mathcal{N}$, to see that $P P_{-}<P<P_{+}$ in $\mathcal{N}_{r}$ and that $\operatorname{dim}\left(P-P_{-}\right)<\infty$; in other words, $K \in\left(\mathcal{A}_{r}\right), \cap \mathcal{K}$ as desired. We use the characterization of $\mathcal{A}_{b}$ given in Theorem 2.4 in two places here - first to tell us that $\operatorname{dist}\left(R A_{n}, \mathcal{A}_{b}\right) \geq \operatorname{dist}\left(R A_{n}, \mathcal{A}_{r}\right)$, which we need to establish the contradictions, and, second, to tell us that the operators $A_{n}$ defined in the third step (showing that $\left.\operatorname{dim}\left(P-P_{-}\right)<\infty\right)$ are in $\mathcal{A}_{b}$ because they are in $\mathcal{A}_{r} \cap \mathcal{K}$. (Note that, because $\left(P-P_{-}\right)$ is now only an atom in $\mathcal{N}_{r}$, these operators need not be in $\mathcal{A}$.)

For the reverse inclusion, it suffices to show that $\left(\mathcal{A}_{r}\right)_{r} \cap \mathcal{K} \subseteq\left(\mathcal{A}_{b}\right)_{b}$. For this, first set

$$
\mathcal{N}_{0}=\left\{P \in \mathcal{N}_{r}: P_{-}<P<P_{+} \text {in } \mathcal{N}_{r} \text { and } \operatorname{dim}\left(P-P_{-}\right)<\infty\right\}
$$

so that $\left(\mathcal{N}_{r}\right)_{r}=\mathcal{N}_{r} \backslash \mathcal{N}_{0}$. Observe that, if $P \in \mathcal{N}_{0}$, then $\operatorname{dim}\left(P_{+}-P\right.$ ) $=\infty$ (as $\mathcal{N}_{r}$ cannot contain adjacent finite-dimensional atoms) and that each atom of $\left(\mathcal{N}_{r}\right)$, can contain at most one element of $\mathcal{N}_{0}$. Indeed, if $(Q-P)$ is an atom in $\left(\mathcal{N}_{r}\right)$, and if $E$ and $F$ in $\mathcal{N}_{0}$ satisfy $P<E<F<Q$, then $\operatorname{dim}(F-E)=\infty$. Therefore, working in $\mathcal{N}_{r}$, we have $P<E<\ldots<F_{2}<F_{1}<F<Q$, where $F_{1}=F_{-}, F_{k+1}=\left(F_{k}\right)_{-}$, and $\operatorname{dim}\left(F_{k}-E\right)=\infty$ for all $k \geq 1$. Thus, there is a strong limit point of $\mathcal{N}_{r}$ lying strictly between $P$ and $Q$, contradicting the fact that $(Q-P)$ is an atom in $\left(\mathcal{N}_{r}\right)_{r}$.

Suppose, then, that $\mathcal{N}_{0}=\left\{E_{i}: i \in \mathcal{Z}\right\}$ and that $\left\{\left(Q_{i}-P_{i}\right): i \in \mathcal{Z}\right\}$ is the corresponding set of atoms in $\left(\mathcal{N}_{r}\right)$, (so $P_{i}<E_{i}<Q_{i}$ for all $i$ ). Note that $P_{i}=\left(E_{i}\right)_{-}$in $\mathcal{N}_{r}$ and, therefore, that $\operatorname{dim}\left(E_{i}-P_{i}\right)<\infty$. Now, given $K \in\left(\mathcal{A}_{r}\right)_{r} \cap \mathcal{K}$, let 


$$
K^{\prime}=\sum_{i \in \mathcal{Z}} \oplus\left(Q_{i}-E_{i}\right) K\left(E_{i}-P_{i}\right)
$$

We have $K^{\prime} \in\left(\mathcal{A}_{r}\right) \cap \mathcal{K}$ and $\left(K-K^{\prime}\right) \in \mathcal{A}_{r} \cap \mathcal{K} \subseteq \mathcal{A}_{b}$. Therefore, $\operatorname{dist}\left(K, \mathcal{A}_{b}\right) \leq \| K-$ $\left(K-K^{\prime}\right)\|=\| K^{\prime} \|$. At the same time, if $R$ is an arbitrary element of $\mathcal{A}_{b}=\mathcal{A}+\left(\mathcal{A}_{r} \cap \mathcal{K}\right)$, then $E_{i}^{\perp} R E_{i}=0$ for all integers $i$ so that $\left(Q_{i}-E_{i}\right)(K-R)\left(E_{i}-P_{i}\right)=\left(Q_{i}-E_{i}\right) K\left(E_{i}-P_{i}\right)$ for all $i$. It follows that

$$
\|K-R\| \geq \sup _{i \in Z}\left\|\left(Q_{i}-E_{i}\right) K\left(E_{i}-P_{i}\right)\right\|=\left\|K^{\prime}\right\|
$$

for all such $R$. Thus, $\operatorname{dist}\left(K, \mathcal{A}_{b}\right)=\inf \left\{\|K-R\|: R \in \mathcal{A}_{b}\right\} \geq\left\|K^{\prime}\right\|$ as well. We conclude that

$$
\operatorname{dist}\left(K, \mathcal{A}_{b}\right)=\left\|K^{\prime}\right\|
$$

for all $K$ in $\left(\mathcal{A}_{\tau}\right), \cap \mathcal{K}$.

Let the sequence $\left\{S_{n}\right\} \subseteq \mathcal{A}_{b}$ converge weak-* to 0 . Then, for each $n$, we have $K S_{n} \in\left(\mathcal{A}_{r}\right), \cap \mathcal{K}$ so that

$$
\begin{aligned}
\operatorname{dist}\left(K S_{n}, \mathcal{A}_{b}\right) & =\left\|\left(K S_{n}\right)^{\prime}\right\| \\
& =\sup _{i \in \mathcal{Z}}\left\|\left(Q_{i}-E_{i}\right) K\left(E_{i}-P_{i}\right) S_{n}\left(E_{i}-P_{i}\right)\right\| .
\end{aligned}
$$

The set of norms $\left\{\left\|S_{n}\right\|\right\}$ is bounded, say by $M$, and $K$ is compact, so, given $\epsilon>0$, there exists a natural number $N$ such that $\left\|\left(Q_{i}-E_{i}\right) K\left(E_{i}-P_{i}\right)\right\|<\epsilon / M$ for all integers $i$ with $|i| \geq N$. Moreover, as $\left(E_{i}-P_{i}\right)$ is finite-dimensional for each $i$ and $S_{n} \rightarrow 0$ weak-*, it follows that $\lim _{n \rightarrow \infty}\left\|\left(E_{i}-P_{i}\right) S_{n}\left(E_{i}-P_{i}\right)\right\|=0$ for each $i$. Thus, for each integer $i$, choose a natural number $n_{i}$ such that $\left\|\left(E_{i}-P_{i}\right) S_{n}\left(E_{i}-P_{i}\right)\right\|<\epsilon /\|K\|$ for all $n \geq n_{i}$. With $m=\max \left\{n_{i}:|i| \leq N\right\}$, we then have $\left\|\left(K S_{n}\right)^{\prime}\right\| \leq \epsilon$ for all $n \geq m$. It follows that $K \in\left(\mathcal{A}_{b}\right)_{b}$ as claimed.

We observed above that $\left(\mathcal{N}_{\infty}\right)_{\infty}=\mathcal{N}_{\infty}$, whence, $\left(\mathcal{A}_{\infty}\right)_{\infty}=\mathcal{A}_{\infty}$. Comparison of Theorems 2.4 and 3.1 then yields the following corollary.

Corollary 3.2. Let $\mathcal{A}=\operatorname{alg} \mathcal{N}$ be the nest algebra corresponding to the nest $\mathcal{N}$. Then $\left(\mathcal{A}_{B}\right)_{B}=\mathcal{A}_{B}$.

A simpler proof of this Corollary on its own is obtained by first using an argument similar to that given in the first part of the proof of Theorem 3.1 to show that $\left(\mathcal{A}_{B}\right)_{B} \subseteq(\mathcal{A}+\mathcal{K}) \cap\left(\mathcal{A}_{\infty}\right)_{\infty}$ and then applying the observation that $\left(\mathcal{A}_{\infty}\right)_{\infty}=\mathcal{A}_{\infty}$ to conclude that $\left(\mathcal{A}_{B}\right)_{B} \subseteq(\mathcal{A}+\mathcal{K}) \cap \mathcal{A}_{\infty}=\mathcal{A}_{B}$. The reverse inclusion is automatic from the definitions.

While we can have $\left(\mathcal{A}_{b}\right)_{b} \neq \mathcal{A}_{b}$ (and ${ }_{b}\left({ }_{b} \mathcal{A}\right) \neq{ }_{b} \mathcal{A}$ ), the next result shows that the inequality can go no farther. 
Proposition 3.3. For $\mathcal{A}=\operatorname{alg} \mathcal{N}$ a nest algebra, we have

(i) $(\mathcal{A})_{b(3)}=(\mathcal{A})_{b(2)}$ and

(ii) $b^{(3)}(\mathcal{A})=_{b^{(2)}}(\mathcal{A})$.

Proof. For the first assertion, an argument similar to that of Theorem 2.1 and the first part of the proof of Theorem 3.1 shows that $(\mathcal{A})_{b^{(3)}} \subseteq(\mathcal{A}+\mathcal{K}) \cap\left(\left(\mathcal{A}_{r}\right)_{r}\right)$. But, as we observed earlier, $\left(\left(\mathcal{A}_{r}\right)_{r}\right)=\left(\mathcal{A}_{r}\right)_{r}$ from which it follows, using Theorem 3.1' (iv), that $(\mathcal{A})_{b(3)} \subseteq(\mathcal{A})_{b(2)}$. The reverse inclusion is automatic.

The proof of the second assertion is similar.

Example 3.4. We conclude with an example for which all nine second-order Bourgain algebras are different and yet which is still reasonably manageable. Consider the nest

$$
\mathcal{N}=\{0,1\} \cup\left\{Q_{n}: n \geq 1\right\} \cup\left\{\left\{P_{k}^{(2 n+1)}: n \geq 0\right\}\right\}
$$

where the projections $Q_{n}$ decrease strongly to 0 with $\operatorname{dim}\left(Q_{2 n-1}-Q_{2 n}\right)<\infty$ and $\operatorname{dim}\left(Q_{2 n}-Q_{2 n+1}\right)=\infty$ for all $n \geq 1$ and $\operatorname{dim}\left(1-Q_{1}\right)=\infty$, and where $\left\{P_{k}^{(4 n+3)}\right\}=$ $\left\{P_{k}^{(4 n+3)}: k \geq 1\right\}$ is an increasing sequence of projections between $Q_{4 n+3}$ and $Q_{4 n+2}$ with $\operatorname{dim}\left(P_{k+1}^{(4 n+3)}-P_{k}^{(4 n+3)}\right)<\infty$ for all $k, \operatorname{dim}\left(P_{1}^{(4 n+3)}-Q_{4 n+3}\right)=\infty$ and $P_{k}^{(4 n+3)} \rightarrow Q_{4 n+2}$ in the strong operator topology as $k \rightarrow \infty$, and where $\left\{P_{k}^{(4 n+1)}\right\}=\left\{P_{k}^{(4 n+1)}: k \geq 1\right\}$ is a decreasing sequence of projections between $Q_{4 n+1}$ and $Q_{4 n}$ with $\operatorname{dim}\left(P_{k}^{(4 n+1)}-P_{k+1}^{(4 n+1)}\right)<\infty$ for all $k, \operatorname{dim}\left(Q_{4 n}-P_{1}^{(4 n+1)}\right)=\infty$ and $P_{k}^{(4 n+1)} \rightarrow Q_{4 n+1}$ in the strong operator topology as $k \rightarrow \infty$. (We interpret $Q_{0}$ as 1 ). Thus, $\mathcal{N}$ has order type $\cdots \oplus \mathcal{Z}_{-} \oplus 1 \oplus \mathcal{Z}_{+} \oplus 1 \oplus \mathcal{Z}_{-}$.

Careful consideration shows that, in this example,

$$
\begin{aligned}
\mathcal{N}_{r} & =\{0,1\} \cup\left\{Q_{n}: n \neq 4 k+3\right\} \cup\left\{P_{1}^{(4 n+3)}\right\}, \\
\mathcal{N}_{l} & =\{0,1\} \cup\left\{Q_{n}: n \neq 4 k\right\} \cup\left\{P_{1}^{(4 n+1)}\right\}, \text { and } \\
\mathcal{N}_{\infty} & =\{0,1\} \cup\left\{Q_{n}: n \geq 1\right\} \cup\left\{P_{1}^{(2 n+1)}\right\} .
\end{aligned}
$$

Further examination then shows that

$$
\begin{aligned}
\left(\mathcal{N}_{r}\right)_{r} & =\{0,1\} \cup\left\{Q_{2 n}: n \geq 1\right\} \cup\left\{P_{1}^{(4 n+3)}\right\}, \\
\left(\mathcal{N}_{r}\right)_{1} & =\{0,1\} \cup\left\{Q_{4 n+1}\right\} \cup\left\{Q_{4 n}\right\} \cup\left\{P_{1}^{(4 n+3)}\right\}, \text { and } \\
\left(\mathcal{N}_{r}\right)_{\infty} & =\mathcal{N}_{r} .
\end{aligned}
$$

Moreover, 


$$
\begin{aligned}
\left(\mathcal{N}_{1}\right)_{r} & =\{0,1\} \cup\left\{Q_{4 n+2}\right\} \cup\left\{Q_{4 n+3}\right\} \cup\left\{P_{1}^{(4 n+1)}\right\}, \\
\left(\mathcal{N}_{1}\right)_{1} & =\{0,1\} \cup\left\{Q_{2 n+1}\right\} \cup\left\{P_{1}^{(4 n+1)}\right\}, \text { and } \\
\left(\mathcal{N}_{1}\right)_{\infty} & =\mathcal{N}_{1} .
\end{aligned}
$$

Finally, we have

$$
\begin{aligned}
\left(\mathcal{N}_{\infty}\right)_{r} & =\{0,1\} \cup\left\{Q_{2 n}\right\} \cup\left\{P_{1}^{(2 n+1)}\right\}, \\
\left(\mathcal{N}_{\infty}\right)_{1} & =\{0,1\} \cup\left\{Q_{2 n+1}\right\} \cup\left\{P_{1}^{(2 n+1)}\right\}, \text { and } \\
\left(\mathcal{N}_{\infty}\right)_{\infty} & =\mathcal{N}_{\infty} .
\end{aligned}
$$

The results above seem quite dependent on the nature of nests and nest algebras as we have used both the linear ordering and the distance formula heavily. Yet Lemma 1.1 applies to any local subalgebra of $\mathcal{L}(\mathcal{H})$. It would be interesting to compute the Bourgain algebra(s) for other examples of local algebras (for instance, S. C. Power has found a nice argument showing that the Fourier Binest algebra coincides with its Bourgain algebra) and, more generally, to characterize algebraically the Bourgain algebras of other classes of algebras such as those with invariant subspace lattice given by the tensor product of two nests or some more general class of completely distributive CSL algebras.

\section{REFERENCES}

1. W. ARveson, Interpolation problems in nest algebras, J. Funct. Anal. 20 (1975), 208-233.

2. J. Bourgain, The Dunford-Pettis property for the ball-algebras, the polydisc-algebras, and the Sobolev spaces, Studia Math. 77 (1984), 245-253.

3. J. A. Cima and R. M. Timoney, The Dunford-Pettis property for certain planar uniform algebras, Michigan Math. J. 34 (1987), 99-104.

4. J. A. Cima, S. Janson, and K. Yale, Completely continuous Hankel operators on $H^{\infty}$ and Bourgain algebras, Proc. Amer. Math. Soc. 105 (1989), 121-125.

5. J. A. ERdos, Operators of finite rank in nest algebras, J. London Math. Soc. 43 (1968), 391-397.

6. T. FAll, W. ARveson, and P. Muhly, Perturbations of nest algebras, J. Operator Theory 1 (1979), 137-150.

7. T. G. Feeman, Nest algebras of operators and the Dunford-Pettis property, Canad. Math. Bull. 34 (1991), 208-214.

8. P. G. Ghatage, S. Sun and D. Zheng, A remark on Bourgain algebras on the disk, Proc. Amer. Math. Soc. 114 (1992), 395-398.

9. P. Gorkin, K. IzUCHI and R. MORTINI, Bourgain algebras of Douglas algebras, Canad. J. Math. 44 (1992), 797-804.

10. K. Izuchi, K. Stroethoff and K. Yale, Bourgain algebras of spaces of harmonic functions, Michigan Math. 41 (1994), 309-322. 
11. A. Kata volos and S. C. Power, The Fourier binest algebra, preprint.

12. C. Laurie and W. E. LongstafF, A note on rank-one operators in reflexive algebras, Proc. Amer. Math. Soc. 89 (1983), 293-297.

Department of Mathematical Sciences

VILLANOVA UNIVERSITY

Villanova, PA 19085

U.S.A. 